\title{
Etude Prospective Du Profil Épidémiologique Des Femmes Enceintes Pré-Éclamptiques En Mauritanie
}

\author{
Mariem Sidi Mohamed \\ Setty Sass \\ Laboratoire de biochimie, Biotechnologie, Environnement et Santé- \\ Université Ibn Tofail. Faculté de sciences, Département de sciences de la \\ vie-Kenitra-Maroc
}

\section{Ahmed Zein}

Université de Sciences de Technologies et de Médecine, et Service Gynécoobstétrique de CHCZ-Mauritanie

\section{Ahmed Lbarae}

Institut nationale de Recherche en Santé Publique (INRSP)-Mauritanie

\section{Abderrazzak Khadmaoui}

laboratoires de génétique et biométrie, Université Ibn Tofail. Faculté de sciences, Département de sciences de la vie-Kénitra-Maroc

\section{Aicha Lrhorfi Benharbit Oumaima \\ Rachid Bengueddour}

Laboratoire de biochimie, Biotechnologie, Environnement et SantéUniversité Ibn Tofail. Faculté de sciences, Département de sciences de la vie-Kenitra-Maroc

Doi: 10.19044/esj.2017.v13n36p124 URL:http://dx.doi.org/10.19044/esj.2017.v13n36p124

\section{Abstract}

Pre-eclampsia is a major public health problem in pregnant women. The objective of our study is to describe the socio-demographic and epidemiological profile of pre-eclampsia women. As a result, we conducted a prospective study in the maternity ward of the CHEIKH ZAYED Hospital Center in Nouakchott, during the period from August 2015 to September 2016. In addition, 360 pregnant women were interviewed, $31 \%(n=112)$ with complications of arterial hypertension. In addition, 50 patients among these hypertensive women were pre-eclampsia, of whom $46 \%$ were between 20 and 30 years of age, $52 \%$ were black and $82 \%$ had no fixed profession. Multiple pre-eclampsia gestures accounted for over half of the cases $(62 \%)$. Nevertheless, contrary to the literature, our outcome results are much more 
alarming, which calls for an adequate and effective intervention to deal with this scourge.

Keywords: Pre-eclampsia-pregnant woman-prospective study-muli gestureimpact

\section{Resume}

La pré-éclampsie représente une cause majeure de la morbidité et mortalité materno-infentille chez la femme enceinte dans les pays du tiers monde. L'objectif de cette étude consiste à décrire le profil sociodémographique et épidémiologique des femmes en stade de prééclampsie en Mauritanie. Nous avons mené une étude prospective et descriptive dans le service de maternité du Centre Hospitalier CHEIKH ZAYED de Nouakchott, durant la période allant de l'aout 2015 au septembre 2016. En somme, 360 femmes enceintes ont été recrutées. L'examen clinique a mis en évidence que $31 \%(n=112)$ des gestantes de la maternité présentent des complications d'hypertensionetque 50 parmi euxen stade de prééclampsie, dont $46 \%$ ayant un âge compris entre 20 et 30 ans, 52\% sont de l'ethnie ; maure noire et $82 \%$ sont sans profession. Les multi-gestes prééclamptiques représentaient plus de la moitié des cas (62\%). Cependantl'incidence de la pré-éclampsiechez les femmes enceintes en milieu hospitalier de Nouakchott semble alarmante par rapport aux données de la littérature. Une action efficienteeturgente paraitnécessaire faire face à ce fléau.

Mots-clés : Pré-éclampsie-femme enceinte-étude prospective-multi-gesteincidence

\section{Introduction}

L'hypertension, durant la grossesse, touche environ 5 à $10 \%$ des femmes enceintes. Elle doit être recherchée de façon systématique lors de chaque consultation prénatale, en raison de ses conséquences parfois redoutables, tant chez la mère que chez le fœtus (Clivaz et al, 2009).

Les troubles hypertensifs au cours de la grossesse, constituent une cause importante de morbidité sévère, d'incapacité à long terme et de mortalité pour les mères comme pour leurs nouveau-nés (O.M.S, 2011).

En Afrique et en Asie, près d'un dixième des décès maternels sont attribuées à des troubles hypertensifs survenant cours de la grossesse (O.M.S, 2013). En Amérique latine, ce chiffre est porté à un quart (O.M.S, 2013). En Mauritanie, la mortalité maternelle est estimée à 550 décès pour 100.000 naissances vivantes. Les complications de l'hypertension artérielle est l'un des principales causes immédiates de décès périnataux (P.N.D.S, 2011). 
Parmi les troubles hypertensifs constituant les complications gravidiques, la pré-éclampsie et l'éclampsie présentant des causes majeures de morbidité, de mortalité maternelle et Néonatale (O.M.S, 2013).

La pré-éclampsie, autrefois appelée toxémie gravidique, est la plus dangereuse de ces affections et se déclare chez environ 2,6\% des femmes enceintes. Les femmes atteintes de pré-éclampsie courent un plus grand risque de décollement placentaire, d'insuffisance rénale aigue, d'hémorragie cérébrale, de coagulation intra vasculaire disséminée, d'œdème pulmonaire, de collapsus cardio-vasculaire et d'éclampsie (Beaulieu, 1994).

La précocité du diagnostic et du traitement est, donc, une nécessité absolue. En effet, un traitement inadéquat ou retardé risque de favoriser l'apparition de complications graves sources de morbi-mortalité maternelle et fœtale. Par contre un diagnostic précoce et une prise en charge thérapeutique multidisciplinaire et rapide ont toutes les chances de reverser la maladie pour la mère et de limiter les conséquences chez le fœetus selon le terme de survenue de la pré-éclampsie (Girardie, 2012).

De nombreuses études ont été consacrées à la prise en charge de la pré-éclampsie; elles montraient qu'un dépistage précoce des gestantes à risque, soumises à une prise en charge correcte permet l'amélioration du pronostic maternel et fœtal.

\section{Materiel et methodes}

\section{Type d'étude}

Il s'agit d'une étude prospective transversale, de la prévalence de la pré-éclampsie chez les femmes enceintes au Centre hospitalier Cheikh Zayed (CHCZ) à Nouakchott. Cette enquête est destinée à toutes les femmes enceintes hospitalisées ayant acceptés de faire partie de l'étude et seules n'ayant pas acceptés ont été exclu.

\section{Déroulement de l'enquête}

Un questionnaire a été confectionné, il comporte donc, des items concernant l'état sanitaire et social des femmes enquêtées, ainsi que tous les examens complémentaires demandés de façon systématique (NFS, Urée, créatininémie, glycémie, uricémie, transaminases, protéinurie des 24 heures),

\section{Analyses statistiques}

Les données collectées sont saisies sur un support Excel, après filtration, elles ont été importées sur support logiciel conçu pour l'exploitation des données (SPSS version 22). Les analyses appliquées sont généralement des fréquences en pourcentage, des analyses multiples comme le test khi-deux d'indépendance ont été choisies Pour la confirmation ou la recherche de liaison. 


\section{Resultats et discussions}

\section{Caractéristiques sociodémographiques des femmes enceintés enquêtées}

L'étude que nous avons menée a portée sur 360 gestantes en cas d'hospitalisation au niveau de la maternité de l'hôpital Cheikh Zayed. La provenance de la majorité de ces femmes était le milieu urbain dans $89,7 \%$ $(n=323)$, et en particulier la zone de Nouakchott contre 10,3\% de milieu rural.Toutefois, l'âge moyen de était de 29,16 $\pm 0,35$ ans, avec un âge minimal de 15 ans et un âge maximal de 47 ans. La distribution s'est montrée logiquement homogène avec un coefficient de variation ne dépassant pas 24\%. Cependant, les valeurs des coefficients d'asymétrie et d'aplatissement confirment l'aspect gaussien de notre distribution, ils sont, respectivement, de 0,199 et $-0,504$.

Néanmoins, la répartition des gestantes en fonction du niveau d'instruction montre; que 20,3\% d'entre eux n'ont jamais été scolarisés, $35,3 \%$ ont été arrivées à un niveau primaire, $14,7 \%$ à un niveau secondaire et $29,7 \%$ avaientun niveau supérieur. En ce qui concerne la profession, $76,94 \%$ de notre échantillons sont des femmes au foyer contre $23,06 \%$ exercent, soit une activé commerciale ou publique dont $8,8 \%$ des étudiantes. Selon l'origine ethnique, les ressortissantes des maures blancs avaient été majoritaire dans notrefile, avec $63 \%$, contre respectivement $33 \%$ et $4 \%$ des ressortissantes des maures noires et de négro-africaines.

\section{Etude du profil épidémiologique de la pré-éclampsie dans notre échantillon}

Dans le cadre du dépistage de la pré-éclampsie, toutes femmes enceintes reçue au niveau de 1 'hôpitalCZ, sera soumise systématiquement à une prise de sa tension systolique et diastolique. Sur la base de ce résultat par rapport au chiffre normal qui est $140 / 90 \mathrm{~mm} \mathrm{Hg}$, les femmes seront réparties en deux groupes : les normo tendues avec un chiffre inférieur ou égal au chiffre normal et les hypertendues pour tous ce qu'est supérieure.

Cette mesure de la tentions artérielle a mis en évidence que $31 \%$ $(n=112)$ des consultantes ont un chiffre supérieur au chiffre normal de la tension artérielle, ce qui le bascule dans le groupe des hypertendue.

Afin de déterminer la prévenance de la pré éclampsie parmi les hypertendues, le critère choisi a été la présence des protéines dans les urines. Le screening des urines des 112 gestantes par des bandelette- test a montré une prévalence de l'ordre de $44,64 \%$ d'entre eux $(n=50)$ ont des protéines dans leurs urines.

Cette prévalence est largement supérieure à seule trouvée par (Harioly et al, 2009), à Antananarivo qui été de 23\% chez 283 femmes âgées de 15 à 45 ans pré-éclamptiques et/ou éclamptiques 


\section{Etude de la liaison entre la pré-éclampsie et certains paramètres sociodémographiques}

Cette partie est consacrée à la rechercher des liens éventuelles entre la variable dépendent qui est l'hypertension et les autres variables indépendantes à savoir : âge, milieu de provenance, niveau d'instruction et la profession et la gestité. Le tableau I, récapitule les principaux résultats.

Tableau I.Étude de la liaison entre la pré-éclampsie et certains variables sociodémographiques.

\begin{tabular}{|c|c|c|c|c|c|c|c|}
\hline \multirow[b]{2}{*}{ Variable } & \multirow[b]{2}{*}{ Modalité } & \multicolumn{2}{|c|}{ Hypertension $(n=112)$} & \multirow{2}{*}{$\begin{array}{c}\text { Tentions } \\
\text { normales } \\
(\mathrm{n}=\mathbf{2 4 8})\end{array}$} & \multirow[b]{2}{*}{ Total (VPP) } & \multirow[b]{2}{*}{$\begin{array}{l}\text { Khi- } \\
\text { deux }\end{array}$} & \multirow[b]{2}{*}{ p-value } \\
\hline & & $\begin{array}{c}\text { Pré- } \\
\text { éclamptique } \\
(\mathbf{n}=\mathbf{5 0})\end{array}$ & $\begin{array}{l}\text { Non Pré- } \\
\text { éclamptique } \\
(n=62)\end{array}$ & & & & \\
\hline \multirow{4}{*}{ Age } & $<20$ ans & $05(10 \%)$ & 05 & 24,0 & $34,0(14,71 \%)$ & \multirow{4}{*}{06,0} & \multirow{4}{*}{$\begin{array}{l}0,43 \\
\text { (ns) }\end{array}$} \\
\hline & $20<>30$ ans & $23(46 \%)$ & 32 & 132 & $187(12,29 \%)$ & & \\
\hline & $30<>40$ ans & $19(38 \%)$ & 18 & 82,0 & $119(15,97 \%)$ & & \\
\hline & $>40$ ans & $03(6 \%)$ & 07 & 10,0 & $20,0(0150 \%)$ & & \\
\hline \multirow{3}{*}{ Ethnie } & Maure noire & $26(52 \%)$ & 26 & 65,0 & $117(22,00 \%)$ & \multirow{3}{*}{27,5} & \multirow{3}{*}{$0,00(\mathrm{~s})$} \\
\hline & Maure blanc & $19(38 \%)$ & 31 & 177 & $227(08,30 \%)$ & & \\
\hline & Négro africaine & $05(10 \%)$ & 05 & 06,0 & $16,0(31,00 \%)$ & & \\
\hline \multirow{2}{*}{ provenance } & Urbain & $43(86 \%)$ & 54 & 226 & $323(13,31 \%)$ & \multirow{2}{*}{1,75} & \multirow{2}{*}{$0,20(\mathrm{~ns})$} \\
\hline & Rural & $07(14 \%)$ & 08 & 22,0 & $37,0(18,91 \%)$ & & \\
\hline \multirow{4}{*}{ Instruction } & non scolarisée & $09(18 \%)$ & 20 & 44,0 & $73,0(12,32 \%)$ & \multirow{4}{*}{9,63} & \multirow{4}{*}{$1,37(\mathrm{~ns})$} \\
\hline & Primaire & $20(40 \%)$ & 21 & 86,0 & $127(15,74 \%)$ & & \\
\hline & Secondaire & $07(14 \%)$ & 04 & 42,0 & $53,0(13,20 \%)$ & & \\
\hline & Supérieur & $14(28 \%)$ & 17 & 76,0 & $107(13,08 \%)$ & & \\
\hline \multirow{2}{*}{ Profession } & Ménager & $41(82 \%)$ & 46 & 190 & $277(14,80 \%)$ & \multirow{2}{*}{8,75} & \multirow{2}{*}{$0,36(\mathrm{~ns})$} \\
\hline & fonctionnaire & $09(18 \%)$ & 16 & 58,0 & $83,0(10,08 \%)$ & & \\
\hline \multirow{3}{*}{ Gestité } & 1 & $11(22 \%)$ & 16 & 60,0 & $87,0(12,64 \%)$ & \multirow{3}{*}{40,96} & \multirow{3}{*}{$\begin{array}{c}0,017 \\
(\mathrm{~s})\end{array}$} \\
\hline & 2 à 3 & $08(16 \%)$ & 14 & 79,0 & $101(07,92 \%)$ & & \\
\hline & Supérieur à 3 & $31(62 \%)$ & 32 & 109 & $172(18,02 \%)$ & & \\
\hline
\end{tabular}

Ns : non significative ; $\mathrm{s}$ : différence significative ; VPP : valeur prédictive positive

\section{- Selon l’âge}

La répartition des femmes enceintes selon l'âge et l'état sanitaire, montre que $84 \%$ des femmes pré-éclamptiques sont âgées entre 20 et 40 ans, dont la VPP atteint 28,26\%. Le test khi-deux d'indépendance ne montre aucun lien entre ces deux facteurs (khi-deux $=6 ; \mathrm{p}<0,43$ ). La tranche d'âge la plus touchée est celle de 20 à 30 ans, dont $15 \%$ sont des cas hypertendus et $37 \%$ ne le sont pas. Selon (Takongmo et al, 2000), cette tranche d'âge 20-30 ans correspond à une période pendant laquelle l'activité génitale de la femme culmine.

\section{- Selon l'origine ethnique}

En Mauritanie, il y a 3 catégories de population les maures (maure= Habitant arabo-berbère du nord de l'Afrique Habitant du Sahara occidental 
vivant principalement en Mauritanie.) noires ; les maures blancs et les negro africains. Le test khi-deux montre une liaison significative entre l'état de santé et l'origine ethnique (khi-deux $=27,5 ; \mathrm{p}<0,000)$. En effet, sur les 50 femmes déclarées pré-éclamptiques, $52 \%$ sont des maures noires, $38 \%$ sont des maures blancs et le reste de cas sont des negro. Cette constatation corrobore les résultats du (Mac Kay, 2001), qui confirment que les afroaméricaines sont touchées par la pré-éclampsie et souffrent de complications materno-foetales plus que les américaines de race blanche.

Cependant, laquasi-totalité des femmes qui souffrent de la prééclamptique sont issues du milieu urbaine $(n=43)$, soit $86 \%$ contre seulement $14 \%$ du milieu rural $(n=7)$. Ces résultats sont similaires à ceux confirmés par (Harioly et al, 2009), Ceci pourrait être expliqué par le fait que les citadines sont beaucoup plus exposées à l'hypertension artérielle fautedes facteurs de risque tels que, le stress, l'absence l'activité physique.

\section{- Selon le niveau d'instruction et la profession}

La répartition des femmes en état d'hypertension pré-éclamptiques selon le niveau d'instruction et la profession, nous montre que $40 \%$ deces patientes ont atteint un niveau primaire, $28 \%$ ont arrivé à un niveau supérieur, et $18 \%$ n'ont avaient été scolarisé. En ce qui concerne la profession, les femmes ménagères ont constitué la majorité avec $82 \%$ des cas alors que les fonctionnaires etles commerçantes occupaient une place très faible. Ce même constat a été confirmé par le (Lankoande et al, 1998), qui a montré que la pré-éclamptiques prédomine chez les femmes sans profession avec $86 \%$. Cette situation est fort probablement liée au niveau socioéconomique de la femme.

\section{- $\quad$ Selon la gestité}

Selon nos résultats, la gestité constitue un facteur de risque de la pré éclampsie, en raison de la forte liaison avec l'état d'hypertension (khideux $=40,9, p=0,017)$. Toutefois, sur l'ensemble des femmes pré-éclamptique $(\mathrm{n}=50), 62 \%$ sont des multi-gestes (supérieur à 3 grossesses), 22\% de ces patientes sont des primi-gestes (une seule grossesse) et 16\% ont développé deux ou trois grossesses (paucigeste). Notre constat par rapport à l'associons entre la multi-gestité et la pré éclampsie a été diffèrent de celui de (Boubacar, 2011) qui a trouvé une forte association avec primigestes de l'ordre de 51\%. Tandis que, (Dao, 2005), (Dramane, 2006), et (Sanogo, 2001), ont signalé une prédominance des paucigestes avec respectivement des taux de $35,3 \% ; 36,4 \%$ et $32,5 \%$. 


\section{Conclusion}

L'étude prospective qui vise a déterminé la prévalence de la prééclampsie chez les femmes enceintes dans les services de maternité du Centre Hospitalier CHEIKH ZAYED à Nouakchott a été effectuée durant la période allant de 1'aout 2015 au septembre 2016.

Cette étude a permis de conclure que :

Une proportion de $31 \%$ femmes enquêtées, ont été hypertendues et que $44,64 \%$ parmi eux sont en pré-éclamptiques, ce qui traduit une prévenance de la pré éclampsie de l'ordre de 44,64\%, parmi ces femmes.Ce pré éclampsie prévalue avec un certain lien d'association chez les gestante sans profession, de la race maure noire issues du milieu urbain par contre notre résultat ne montre pas un lien entre cette pathologie et le niveau d'instruction des gestantes.

Cette étude assez sommaire, trouve son enrage logique dans le cadre du consensus national et international de la lutte contre la morbidité et la mortalité maternelle. Son résultat peut servir comme un plaidoyer pour les décideurs, afin de renforcer d'avantage les efforts de sensibilisation et des répercutions des problèmes liés à la pré-éclampsie en Mauritanie.

\section{References:}

1. Dao S.Z HTA/grossesse à propos de 120 cas à l'HGT en 2004. Thèse de médecine Bamako 2005, $\mathrm{n}^{0} 98$.

2. Dramane N.F.HTA et Grossesse à propos de 85 cas au Centre de référence de la commune II du district de Bamako en 2005.Thèse de médecine Bamako 2006, $\mathrm{n}^{0} 23$.

3. L. ClivazMariotti P. Saudan R. Landau Cahana A. Pechère-Bertschi . Hypertension chez la femme enceinte, 2009,Revue Médicale Suisse.

4. Lankoande J, Toure B, Ouedraogo A, Ouedraogo C.M.R, Ouatta-ra $\mathrm{T}$, Bonate $\mathrm{B}$, Kone $\mathrm{B}$. Les éclampsies àla maternité du Centre Hospitalier universitaire Yalgado OUEDRAOGO de Ouagadou-gou. Med. Afr. Noire 1998, 45(6): 339-402.

5. M,D. Beaulieu,Prévention de la pré-éclampsie, 1994,158.

6. M.Boubacar Djan Diallo,Hypertension artérielle sur grossesse à l'hôpital NianankoroFomba de Ségou, 2011-2012,88

7. M.O.J HariolyNirina, T.Y Rasolonjatovo, M Andrianirina, D.M.A Randriambololona , D.B Ranoaritiana , J.J Andrianjatovo , J.M Randriamiarana, Profil épidémiologique des pré-éclampsies et des éclampsies admises à la réanimation des adultes de la maternité de Befelatanana, Revue d'Anesthésie-Réanimation et de Médecine d'Urgence 2009(July-August); 1(3): 22-24.

8. Mac Kay AP, Berg CJ, Atrash HK. Pregnancy-related mortality from pree-clampsia and eclampsia. ObstetGynecol 2001;97:533Ŕ8. 
9. OMS, Prévention et traitement de la pré-éclampsie et de l'éclampsie, résumé des recommandations, 2011.

10. OMS, Recommandations de l'OMS pour la Prévention et le Traitement de la Pré-éclampsie et de l'éclampsie-Implications et actions, 2013.

11. P.Girardie, Pré-éclampsie, chapitre 622012,2 (Dr Patrick Girardie UADM - Pôle de Réanimation - Service de Réanimation Polyvalente - Hôpital Roger Salengro - CHRU).

12. Processus d'élaboration du Plan National De Développement Sanitaire (2012-2020),rapport d'analyse de situation du secteur de la santé en Mauritanie, $2011,31$.

13. Sanogo A :HTA / grossesse au CSREF C_IV Thèse de médicine Bamako 2001, $n^{0} 44$.

14. Takongmo $\mathrm{S}$ et al. (2000) Takongmo S, Binam F, Simeu CH, Ngassa $\mathrm{P}$, Kouam L, Malonga $\mathrm{E}$. Aspects thérapeutiques des péritonites génitalesau CHU de Yaounde (Cameroun). Méd Afrique Noire. 2000; 47(1):19-21. 